\title{
Successful treatment with lenalidomide for relapsed adult T-cell leukemia/ lymphoma after cord blood cell transplantation
}

\author{
Katsumichi Fujimaki ${ }^{1}$, Yuto Hibino ${ }^{1}$, Kumiko Kishimoto ${ }^{1}$, Shinichiro Watanabe ${ }^{2}$, Satoshi Koyama ${ }^{3}$, Taiki Ando ${ }^{3}$, \\ Heiwa Kanamori ${ }^{3}$, Hideaki Nakajima ${ }^{4}$
}

${ }^{1}$ Department of Hematology, Fujisawa City Hospital, Kanagawa, Japan, ${ }^{2}$ Department of Clinical Laboratory Medicine, Fujisawa City Hospital, Kanagawa, Japan, ${ }^{3}$ Department of Hematology, Kanagawa Cancer Center, Kanagawa, Japan, ${ }^{4}$ Department of Hematology and Clinical Immunology, Yokohama City University School of Medicine, Kanagawa, Japan

\section{Abstract}

The prognosis for relapsed adult T-cell leukemia/lymphoma (ATL) after allogeneic hematopoietic stem cell transplantation is poor. Here, we report the case of a 67-year-old man who survived for 26 months after treatment with lenalidomide for post-transplant relapsed ATL. He underwent induction therapy with two cycles of modified VCAP-AMP-VECP and achieved complete remission. He received cord blood cell transplantation following a reduced-intensity conditioning regimen. Seven months after transplantation, swelling of the systemic lymph nodes appeared, and relapsed ATL was diagnosed based on a biopsy of the cervical lymph node. Treatment with $10 \mathrm{mg}$ of lenalidomide induced partial remission. At 18 months after transplantation, skin tumors were successfully treated by increasing the dose of lenalidomide to $15 \mathrm{mg}$ with the emergence of skin graft-versus-host disease. Although he died from ATL at 34 months after transplantation, systemic relapsed lesions were controlled by treatment with lenalidomide for 26 months. Our case suggests that lenalidomide is well tolerated and is an effective option for the treatment of post-transplant relapsed ATL.

Key words: lenalidomide, adult T-cell leukemia/lymphoma, allogeneic hematopoietic stem cell transplantation

Submitted November 25, 2020; Accepted March 2, 2021; Published online April 30, 2021; Issued online May 25, 2021

Correspondence: Katsumichi Fujimaki, Department of Hematology, Fujisawa City Hospital, 2-6-1 Fujisawa, Fujisawa City, Kanagawa 2518550, Japan, E-mail: kkfujimaki@gmail.com

\section{Introduction}

Allogeneic hematopoietic stem cell transplantation (allo-HCT) is the only curative therapy for patients with aggressive adult T-cell leukemia/lymphoma (ATL). However, approximately $40 \%$ of patients who receive allo-HCT experience relapse ${ }^{1}$. There are several treatment options for relapsed ATL, including chemotherapy, immunosuppressive agent withdrawal, donor lymphocyte infusion (DLI) from the original donor, or a second alloHCT; however, the prognosis of patients with relapse is not promising with a median survival time of 4-6 months after relapse ${ }^{2,3}$. Lenalidomide induces antitumor immune responses mediated by $\mathrm{T}$ and $\mathrm{NK}$ cells ${ }^{4}$. Although lenalidomide has been approved for patients with ATL in Japan, clinical information on its efficacy and safety in post-transplant relapsed ATL is limited ${ }^{5-7}$. There have been only three reports of successful treatment. Ando et al. reported a patient who was successfully treated with lenalidomide ${ }^{5}$. Morishige et al. reported a patient who was successfully treated with sequential mogamulizumab and lenalidomide ${ }^{6}$. Sakamoto et al. reported four patients who received lenalidomide; two patients achieved a complete response ${ }^{7}$. Here, we report the case of an elderly patient who survived for 26 months after treatment with lenalidomide for post-transplant relapsed ATL.

\section{Case Presentation}

A 67-year-old man was referred to our hospital for hypercalcemia. Computed tomography revealed systemic lymph node swelling. His laboratory data were as fol- 


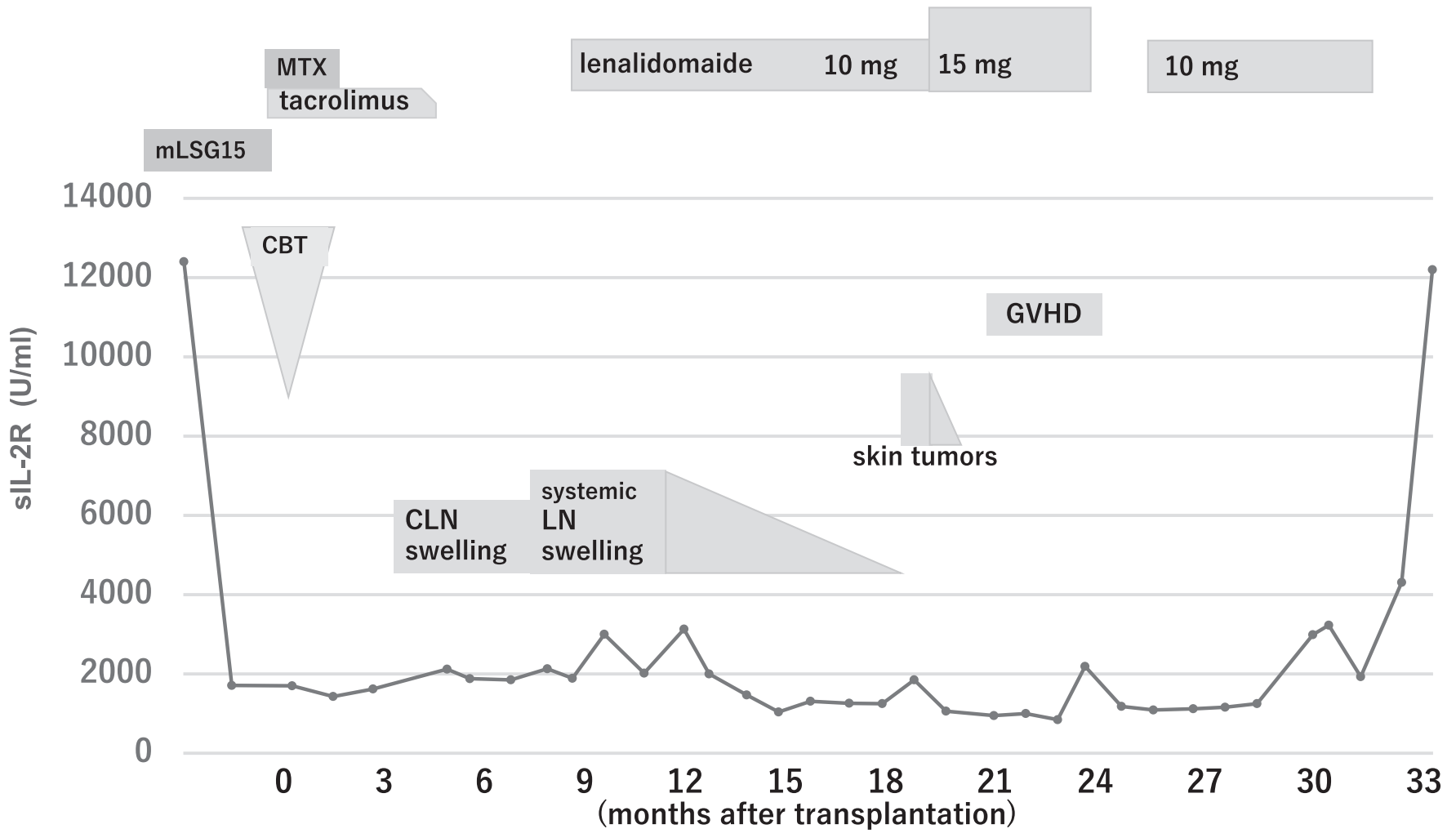

Figure 1. Clinical course

mLSG15, modified VCAP-AMP-VECP; CLN, cervical lymph node; LN, lymph node; MTX, methotrexate.

lows: white blood cell count, $13.9 \times 10^{9} / \mathrm{L}$ (including $18 \%$ abnormal lymphocytes, $71 \%$ neutrophils, $5.5 \%$ lymphocytes, and 5\% monocytes); platelet count, $364 \times 10^{9}$ / $\mathrm{L}$; hemoglobin, $11.2 \mathrm{~g} / \mathrm{dL}$; serum calcium, $16.3 \mathrm{mg} / \mathrm{dL}$; soluble IL-2 receptor, $12,400 \mathrm{U} / \mathrm{mL}$. Western blotting was positive for HTLV-1 antibodies. Bone marrow examination showed the infiltration of monomorphic small to medium-sized abnormal lymphocytes, which were positive for $\mathrm{CD} 2, \mathrm{CD} 3, \mathrm{CD} 4$ and $\mathrm{CD} 5$ and negative for CD7 and CD8. Based on these findings, acute ATL was diagnosed. He underwent two cycles of modified VCAPAMP-VECP $^{8}$ and achieved complete remission. Because he had no HLA-matched related donors and early transplantation was required, he received cord blood cell transplantation $(\mathrm{CBT})$ with $3.3 \times 10^{7} / \mathrm{kg}$ nucleated cells. A reduced-intensity conditioning regimen comprising fractionated total body irradiation (4 Gy in two fractions), $125 \mathrm{mg} / \mathrm{m}^{2}$ fludarabine, and $80 \mathrm{mg} / \mathrm{m}^{2}$ melphalan was administered; tacrolimus and short-term methotrexate were used for graft-versus-host disease (GVHD) prophylaxis (Figure 1). Three months after transplantation, positron emission tomography/computed tomography (PET-CT) revealed cervical lymph nodes swelling. Relapsed ATL was clinically suspected. Tacrolimus was discontinued to enhance the graft-versus-ATL effect. No obvious enlargement of the cervical lymph nodes was noted for some time, suggesting a graft-versus-ATL effect. However, PET-CT revealed systemic lymph node swelling 7 months after transplantation. Relapsed ATL was diagnosed based on cervical lymph node biopsy. Immunostaining of tumor cells was negative for $\mathrm{CC}$ chemokine receptor $4(\mathrm{CCR} 4)$. Lenalidomide $(10 \mathrm{mg})$ was administered daily. The systemic lymph nodes were observed to shrink, and the soluble IL-2 receptor level was gradually decreased without GVHD onset. Partial remission was achieved after lenalidomide administration for 8 months. At 18 months after transplantation, the patient was presented with skin tumors on his neck and back. Although no biopsies of the tumors were performed, ATL recurrence was suspected. The lenalidomide dose was increased to $15 \mathrm{mg}$. Skin lesions were reduced after 2 weeks. Two months later, the skin on his limbs showed lichen planus-like features. Analysis of lymphocyte subsets in the peripheral blood revealed the significantly increased ratio of $\mathrm{CD} 16^{+} / \mathrm{CD} 57^{-}$and $\mathrm{CD} 8^{+} /$ $\mathrm{CD}_{1} 1 \mathrm{~b}^{+}$cells (Supplementary Figure S1). At 22 months after transplantation, watery diarrhea and fever were noted. Lenalidomide was discontinued because GVHD was suspected; however, no pathological examination was performed. After 2 weeks, his diarrhea and fever resolved. At 25 months after transplantation, lenalidomide was restarted at $10 \mathrm{mg}$. At 32 months after transplantation, his laboratory data showed hypercalcemia and elevated soluble IL-2 receptors with abnormal 
Table 1. Reported cases of treatment of lenalidomide for relapsed ATL after allo-HCT

\begin{tabular}{|c|c|c|c|c|c|c|c|c|}
\hline Case & References & & Dose of Len & $\begin{array}{c}\text { Duration of } \\
\text { treatment }\end{array}$ & Effectiveness & $\begin{array}{c}\text { Reason to discontinue } \\
\text { treatment }\end{array}$ & $\begin{array}{c}\text { PFS after } \\
\text { Len (months) }\end{array}$ & $\begin{array}{c}\text { OS after } \\
\text { Len (months) }\end{array}$ \\
\hline 1 & Ando & [5] & $5 \mathrm{mg} / \mathrm{day}$ & 1 week & CR & Relapse of GVHD & $4+$ & $4+$ \\
\hline 2 & Morishige & {$[6]$} & 15 mg/day & 2 weeks & CR & Progression of GVHD & $7+$ & $7+$ \\
\hline 3 & Sakamoto & {$[7]$} & 10 mg/day & 1 week & CR & Relapse of GVHD & $20+$ & unknown \\
\hline 4 & Sakamoto & {$[7]$} & $5 \mathrm{mg}$, alternate day & 29 weeks & $\mathrm{SD}$ & No & $7+$ & unknown \\
\hline 5 & Sakamoto & {$[7]$} & $5 \mathrm{mg}$, alternate day & 18 weeks & CR & Loss of CR in ATL & 4 & unknown \\
\hline 6 & Sakamoto & {$[7]$} & 15 mg/day & 1 week & PD & $\begin{array}{l}\text { No response to treatment } \\
\text { and progression of GVHD }\end{array}$ & 0 & unknown \\
\hline 7 & Present case & & 10 mg/day & 97 weeks & PR & Progression of ATL & 10 & 26 \\
\hline
\end{tabular}

ATL, adult T-cell leukemia/lymphoma; allo-HCT, allogeneic hematopoietic stem cell transplantation; Len, lenalidomide; PFS, progressionfree survival; OS, overall survival; CR, complete response; SD, stable disease; PD, progressive disease; PR, partial response; GVHD, graft versus host disease.

lymphocytes. He died 34 months after transplantation, and the cause of death was relapsed ATL. He survived for 26 months after treatment with lenalidomide for posttransplant relapsed ATL.

\section{Discussion}

The graft-versus-ATL effect is known to play an important role in preventing relapse. Itonaga et al. analyzed 35 patients with post-transplant relapsed ATL; the median survival times after relapse or progression were 16.9 and 3.9 months in the DLI and non-DLI groups, respectively ${ }^{2}$. Kato et al. also analyzed 252 patients with post-transplant relapsed ATL; compared with those without DLI, 36 patients with DLI had better survival outcomes $^{3}$. DLI appears to be one of the most effective therapies for post-transplant-relapsed ATL. To enhance the graft-versus-ATL effect in patients with post-transplant relapsed ATL after CBT, mogamulizumab and lenalidomide are possible choices ${ }^{3}$. In our case, because tumor cells were negative for CCR4, we selected lenalidomide.

The recommended starting dose of lenalidomide for post-transplant relapse after allo-HCT is not obvious. In patients with relapsed myeloma after allo-HCT, lenalidomide therapy at $10 \mathrm{mg} /$ day may not be feasible because of the induction of severe $\mathrm{GVHD}^{4}$. However, another study showed that lenalidomide therapy at $10 \mathrm{mg} /$ day may be safely administered with acceptable tolerability ${ }^{5}$. In our case, we used lenalidomide treatment at $10 \mathrm{mg} /$ day, referring the starting dose of the treatment for posttransplant relapsed ATL (Table 1). The swelling of the systemic lymph nodes was resolved using $10 \mathrm{mg}$ of lenalidomide, and partial remission was achieved. When skin tumors appeared, we increased the dose from $10 \mathrm{mg}$ to $15 \mathrm{mg}$. The skin tumors disappeared; however, lichen planus-like features appeared on the skin. Analysis of lymphocyte subsets in the peripheral blood revealed the significantly increased ratio of $\mathrm{CD} 16^{+} / \mathrm{CD}^{-} 7^{-}$and $\mathrm{CD}^{+} / \mathrm{CD} 11 \mathrm{~b}^{+}$cells at this time compared with 6 months after transplantation before lenalidomide administration. $\mathrm{CD} 16^{+} / \mathrm{CD} 57^{-} \mathrm{NK}$ cells are terminally mature NK cells, which are enriched especially in the cord blood $^{9}$. de Witte et al. reported that the potential kinetics of terminally mature NK cells reduced the relapse rate after allo-HCT ${ }^{10}$. Christensen et al. showed that CD11b expression on $\mathrm{CD}^{+} \mathrm{T}$ cells may define a subset of recently activated cells ${ }^{11}$. Increased $\mathrm{CD}^{+} / \mathrm{CD} 11 \mathrm{~b}^{+}$levels may reflect the development of chronic GVHD. It was reported that higher $\mathrm{CD} 16^{+} / \mathrm{CD}^{-} 7^{-}$and $\mathrm{CD} 8^{+} /$ $\mathrm{CD} 1 \mathrm{~b}^{+}$lymphocyte levels correlated with reduced disease relapse and a higher incidence of chronic GVHD in hematological malignant diseases after allo-HCT ${ }^{12}$. The activation of NK and T cells was also associated with a clinical response to lenalidomide treatment in patients with relapsed myeloma after allo- $\mathrm{HCT}^{4}$. Kato et al. showed that the skin is a target organ susceptible to the graft-versus-ATL effect ${ }^{3}$. We speculate that lenalidomide might induce a graft-versus-ATL reaction through CD $16^{+} / \mathrm{CD} 7^{-}$lymphocyte expansion and a GVHD reaction through $\mathrm{CD} 8^{+} / \mathrm{CD} 11 \mathrm{~b}^{+}$lymphocyte expansion. Because lymphocyte subset analysis was not performed when $10 \mathrm{mg}$ of lenalidomide was administered, no clear conclusion could be drawn. As shown in Table 1, Ando et al. and Morishige et al. reported GVHD with a graft-versus-ATL reaction following lenalidomide treatment ${ }^{5,6}$. Sakamoto et al. also reported that three of four patients developed GVHD and that two patients achieved complete remission ${ }^{7}$. Lenalidomide could be promising for post-transplant relapsed ATL; however, we should be aware of its potential for inducing GVHD development.

Survival for more than 2 years after treatment with lenalidomide for post-transplant relapsed ATL was not observed previously (Table 1). Although some patients survived over 2 years following relapse after tapering and withdrawal of immunosuppressive agents without $\mathrm{DLI}^{3}$, this is the first report to describe a patient with posttransplant relapsed ATL who survived for more than 2 years after lenalidomide treatment. 
In conclusion, lenalidomide administration is well tolerated and effective; however, care should be taken as this strategy may result in GVHD recurrence. Examining a large number of cases of treatment with lenalidomide for post-transplant relapsed ATL would help clarify its role. Lenalidomide treatment may be useful for relapse after CBT, in which DLI is not available.

\section{Author Contributions}

K. F. and H. N. wrote the manuscript. Y. H., K. K., S. W., S. K., T. A., and H. K. collected the data. All authors read and approved the final manuscript.

\section{Conflicts of Interest}

The authors declare no conflict of interest. Disclosure forms provided by the authors are available here.

\section{References}

1. Hishizawa M, Kanda J, Utsunomiya A, Taniguchi S, Eto T, Moriuchi Y, et al. Transplantation of allogeneic hematopoietic stem cells for adult T-cell leukemia: a nationwide retrospective study. Blood. 2010; 116: 1369-76.

2. Itonaga $\mathrm{H}$, Tsushima $\mathrm{H}$, Taguchi J, Fukushima T, Taniguchi $\mathrm{H}$, Sato S, et al. Treatment of relapsed adult T-cell leukemia/lymphoma after allogeneic hematopoietic stem cell transplantation: the Nagasaki Transplant Group experience. Blood. 2013; 121: 219-25.

3. Kato K, Uike N, Wake A, Yoshimitsu M, Tobai T, Sawayama Y, et al. The outcome and characteristics of patients with relapsed adult $\mathrm{T}$ cell leukemia/lymphoma after allogeneic hematopoietic stem cell transplantation. Hematol Oncol. 2019; 37: 54-61.

4. Wolschke C, Stübig T, Hegenbart U, Schönland S, Heinzelmann M, Hildebrandt Y, et. al. Postallograft lenalidomide induces strong NK cell-mediatedantimyeloma activity and risk for T cell-mediated GvHD: Results from a phase I/II dosefinding study. Exp Hematol. 2013; 41: 134-42.
5. Ando T, Kojima K, Sano H, Kidoguchi K, Kusaba K, Yoshimura M, et al. Successful treatment of post-transplant relapsed adult $\mathrm{T}$ cell leukemia after cord blood transplantation with low-dose, short-term lenalidomide. Leuk Lymphoma. 2018; 59: 2474-7.

6. Morishige S, Nishi M, Saruta H, Arakawa F, Yamasaki Y, Oya S, et al. Complete response following toxic epidermal necrolysis in relapsed adult $\mathrm{T}$ cell leukemia/lymphoma after haploidentical stem cell transplantation. Int J Hematol. 2019; 110: 506-11.

7. Sakamoto H, Itonaga H, Sawayama Y, Furumoto T, Fujioka M, Chiwata M, et al. Treatment with mogamulizumab or lenalidomide for relapsed adult T-cell leukemia/lymphoma after allogeneic hematopoietic stem cell transplantation: The Nagasaki transplant group experience. Hematol Oncol. 2020; 38: 16270.

8. Tsukasaki K, Utsunomiya A, Fukuda H, Shibata T, Fukushima T, Takatsuka Y et al. VCAP-AMP-VECP compared with biweekly CHOP for adult T-cell leukemia-lymphoma: Japan Clinical Oncology Group Study JCOG9801. J Clin Oncol. 2007; 25: 5458-64.

9. Danby R, Rocha V. Improving engraftment and immune reconstitution in umbilical cord blood transplantation. Front Immunol. 2014; 5: 68.

10. de Witte MA, Sarhan D, Davis Z, Felices M, Vallera DA, Hinderlie P, et al. Early Reconstitution of NK and $\gamma \delta$ T Cells and Its Implication for the Design of Post-Transplant Immunotherapy. Biol Blood Marrow Transplant. 2018; 24: 1152-62.

11. Christensen JE, Andreasen SO, Christensen JP, Thomsen AR. $\mathrm{CD} 11 \mathrm{~b}$ expression as a marker to distinguish between recently activated effector CD8 $(+) \mathrm{T}$ cells and memory cells. Int Immunol. 2001; 13: 593-600.

12. Ando T, Tachibana T, Tanaka M, Suzuki T, Ishiyama Y, Koyama $S$, et. al. Impact of graft sources on immune reconstitution and survival outcomes following allogeneic stem cell transplantation. Blood Adv. 2020; 4: 408-19.

https://doi.org/10.31547/bct-2020-018

Copyright (C) 2021 APBMT. All Rights Reserved. 